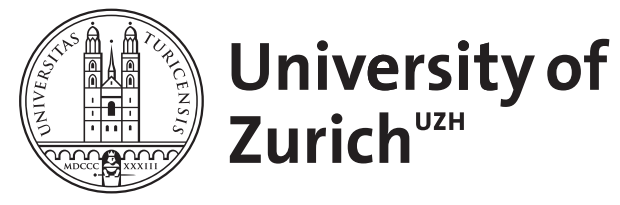

\title{
Gastro-Highlights 2007
}

Schöpfl, R ; Schwizer, W ; Müllhaupt, B ; Rösch, T ; Schachschal, G ; Bauerfeind, P ; Wirth, H P ; Rogler, G ; Fried, M

DOI: https://doi.org/10.1007/s11377-007-0142-1

Posted at the Zurich Open Repository and Archive, University of Zurich ZORA URL: https://doi.org/10.5167/uzh-156722

Journal Article

Published Version

Originally published at:

Schöpfl, R; Schwizer, W; Müllhaupt, B; Rösch, T; Schachschal, G; Bauerfeind, P; Wirth, H P; Rogler, G; Fried, M (2008). Gastro-Highlights 2007. Der Gastroenterologe, 3(1):68-70.

DOI: https://doi.org/10.1007/s11377-007-0142-1 
Gastroenterologe 2008 · 3:68-70 DOI 10.1007/s11377-007-0142-1

Online publiziert: 9. Januar 2008

○) Springer Medizin Verlag 2008

\author{
R. Schöpfl · W. Schwizer · B. Müllhaupt · T. Rösch • G. Schachschal · P. Bauerfeind • \\ H.P. Wirth · G. Rogler · M. Fried \\ Klinik für Gastroenterologie und Hepatologie, Universitätsspital Zürich
}

\title{
Gastro-Highlights 2007
}

\section{Hepatologie}

Zum Langzeitverlauf der nicht alkoholtoxisch bedingten Lebererkrankung zeigte eine schwedische Kohortenstudie mit 129 Patienten, dass nach einer mittleren Nachbeobachtungsdauer von 13,7 Jahren die Mortalität im Falle einer Steatose im Vergleich zu einem Kontrollkollektiv nicht erhöht war, wogegen im Falle einer nicht alkoholisch bedingten Steatohepatitis das Überleben - vor allem wegen kardiovaskulärer oder hepatischer Ereignissen - signifikant verkürzt war. Nach einer USamerikanischen prospektiven Studie besitzt ein Index gebildet aus mittlerem korpuskulären Volumen, dem Verhältnis der Aspartat- und Alanin-Aminotransferasen, Körpermassenindex und Geschlecht bei der Unterscheidung zwischen einer alkoholtoxisch und einer nichtalkoholtoxisch bedingten Lebererkrankung eine höhere Zuverlässigkeit als die herkömmlichen Biomarker.

Gemäß einer retrospektiven Studie zum Verlauf der chronischen Hepatitis B in der Immuntoleranzphase fand bei 12 von 31 Patienten in einem medianen Alter von 30,7 Jahren ein Toleranzverlust statt, wobei 6 Patienten einen schnellen Übergang in einen inaktiven Trägerzustand erfuhren, 3 eine chronische Hepatitis entwickelten und 3 einen vorübergehenden Anstieg der Alanin-Aminotransferasespiegel zeigten. Entgegen den Erwartungen einer niedrigen Seroclearancerate betrug die kumulative Wahrscheinlichkeit für eine HBs-Ag-Seroclearance in einer Studie aus Taiwan mit 1965 Hepatitis-B-Antikörper-positiven asymptomatischen erwachsenen Trägern nach 25 Jahren immerhin $44,7 \%$, während nur $21,5 \%$ der Patienten nach 20 Jahren eine Reaktivierung erfahren hatten. Zur Behandlung der chronischen Hepatitis B mit den neuen Nukleosidanaloga zeigte eine doppelblinde Multicenterstudie mit 1267 Patienten, dass unter der Therapie mit Telbivudin bei einem signifikant größeren Anteil der Patienten eine virologische und biochemische Verbesserung eintrat als unter der Behandlung mit Lamivudin.

Nach einer retrospektiven Studie zum klinischen Verlauf des M. Wilson wurde bei den Patienten mit vornehmlich neuropsychiatrischen Symptomen gegenüber denjenigen mit hepatischen Symptomen eine spätere Manifestation der Symptome, eine längere Zeitspanne vom Symptomauftritt bis zur definitiven Diagnose sowie ein selteneres Ansprechen auf die Behandlung festgestellt. Außerdem ergab eine retrospektive multinationale Studie, dass die Symptome bei 3,8\% der Patienten mit einem M. Wilson erst in einem Alter über 40 Jahre auftraten.

\section{Pankreaserkrankungen}

Bezüglich der Beurteilung des für den Therapieentscheid wichtigen Malignitätsrisikos von Pankreaszysten zeigte eine retrospektive Studie mit 539 Patienten, dass die Pankreaszysten bei 170 Patienten primär reseziert wurden. Dabei wurden vor allem große, symptomatische und solide Zysten entfernt, die sich in $18 \%$ der Fälle tatsächlich auch als maligne erwiesen. Demgegenüber betrug das Malignitätsrisiko der Pankreaszysten bei den 369 nichtoperierten Patienten nach einer Nachbeobachtungsdauer von 24 Monaten lediglich $3 \%$, was in einem ähnlichen Bereich liegt wie die in einer anderen retrospektiven Studie für die Resektion von Pankreaszysten ermittelte postoperative Mortalität von 1,8\%.

Nach einer retrospektiven Analyse von 1000 in den USA von einem einzigen, extrem erfahrenen Chirurgen vorgenommenen Pankreatikoduodenektomien konnten die mittlere Operationsdauer, der postoperative Krankenhausaufenthalt und die postoperative Mortalität im Verlauf der Jahre 1969 bis 2003 deutlich gesenkt werden. Der Eingriff ging bei den Patienten mit einem Pankreaskarzinom ohne Randinfiltration und ohne Lymphknotenbefall einer Fünfjahresüberlebensrate von $41 \%$ einherg und stellt damit eine effektive Behandlungsoption dar. Die im Anschluss an eine Ro- oder R1-Resektion eines Pankreaskarzinoms durchgeführte adjuvante Chemotherapie mit Gemcitabin bewirkte in einer offenen Multicenterstudie mit 368 Patienten nach einer medianen Nachbeobachtungsdauer von $53 \mathrm{Mo}$ naten im Vergleich zur alleinigen Beobachtung eine signifikante Verlängerung der medianen krankheitsfreien Überlebenszeit, was aber keine Auswirkungen auf die Gesamtüberlebenszeit hatte.

Bezüglich der diagnostischen Zuverlässigkeit der Endosonographie ergab eine prospektive Studie mit 31 Patienten, dass diese Methode für die Beurteilung des Schweregrads einer akuten Pankreatitis im Vergleich zum Goldstandard, kontrastmittelverstärkte Computertomographie, eine Sensitivität von $82 \%$ und eine Spezifität von $89 \%$ besitzt. Darüber hinaus konnten mit der Endosonographie gemäß einer auf 689 Patienten mit einer akuten biliären Pankreatitis oder einem Verdacht auf eine biliäre Pankreatitis basierenden Metaanalyse - die Gallengangsteine gegenüber der endoskopisch-retrograden Cholangiopankreatikographie mit einer Sensitivität von $92 \%$ und einer Spezifität von $98 \%$ nachgewiesen werden.

\section{Gastrointestinale Endoskopie und Onkologie}

Zum Nutzen der endoskopischen duktalen Dekompressionstherapie bei der 
chronischen Pankreatitis zeigte eine große deutsche Multicenterstudie mit 1018 ausgewerteten Patienten, dass nach einer mittleren Nachbeobachtungsdauer von 4,9 Jahren $65 \%$ ohne Notwendigkeit eines zusätzlichen chirurgischen Eingriffs schmerzfrei waren. Außerdem ergaben zwei weitere randomisierte Studien, dass bei Patienten mit einer schmerzhaften chronischen obstruktiven Pankreatitis zwei bzw. fünf Jahre nach dem chirurgischen Eingriff bei einem größeren Anteil ein deutlicherer (oder sogar vollständiger) Rückgang der Schmerzen verzeichnet wurde als nach der endoskopischen Therapie.

Bezüglich der diagnostischen $\mathrm{Zu}$ verlässigkeit des Narrowband-Imaging zeigte eine prospektive Studie mit $102 \mathrm{~Pa}$ tienten mit einem oropharyngealen $\mathrm{Tu}$ mor, dass mit diesem neuen endoskopischen Untersuchungsverfahren ösophageale Zweitkarzinome mit einer ähnlichen Treffsicherheit nachgewiesen werden konnten wie mit der Chromoendoskopie unter Verwendung von Lugol-Lösung. Des Weiteren wurden in einer niederländischen Studie die bei 20 Patienten mit einem Barrett-Ösophagus vorhandenen 28 hochgradigen Dysplasien mit Hilfe der Video-Autofluoreszenz mit einer Treffsicherheit von 100\% entdeckt, wobei die relativ hohe Anzahl der falsch-positiven Resultate von 40\% dank dem anschließenden Narrowband-Imaging auf $10 \%$ gesenkt werden konnte.

Da bei einem beträchtlichen Anteil der Pankreaskarzinome eine prognostisch ungünstige HER1-Überexpression vorhanden ist, wurde in einer Doppelblindstudie mit 569 Patienten mit fortgeschrittenem oder metastasierendem Pankreaskarzinom der Nutzen des zusätzlich zu Gemcitabin verabreichten HER1/EGFRTyrosinkinaseinhibitors Erlotinib untersucht. Die Kombinationstherapie mit Erlotinib und Gemcitabin führte gegenüber der Behandlung mit Placebo und Gemcitabin zu einer geringen, aber signifikanten Verlängerung der progressionsfreien und der Gesamtüberlebenszeit. Diese Verbesserung der Prognose muss aber angesichts der erhöhten Inzidenz von Nebenwirkung relativiert werden. Zur Behandlung des fortgeschrittenen hepatozellulären Karzinoms ist mit dem Multikinaseinhibitor
Sorafenib erstmals eine wirksame Chemotherapie verfügbar, die in einer Multicenterstudie mit 602 Patienten im Vergleich zu Placebo eine signifikante Erhöhung der Überlebensrate um 44\% bewirkte.

\section{Reizdarm und kolorektales Karzinom}

Zur präventiven Wirksamkeit der Koloskopie ergab eine US-amerikanische Studie, dass durch die koloskopische Polypektomie die Mortalität an kolorektalen Karzinomen nach einer mittleren Nachbeobachtungsdauer von 14 Jahren gegenüber einem Kontrollkollektiv um 60-75\% gesenkt werden konnte. Darüber hinaus wurde in einer kanadischen Studie bei den Personen, bei denen die Koloskopie einen negativen Befund ergeben hatte, nach einer Nachbeobachtungsdauer von 10 Jahren im Vergleich zur allgemeinen Bevölkerung eine um $72 \%$ verringerte Inzidenz kolorektaler Karzinome festgestellt. Anlässlich der Screeningkoloskopie wurde gemäß zwei umfangreichen Studien mit 50.149 und 5597 Teilnehmern in der Gruppe der 50- bis 66-Jährigen in 5,7 bzw. 8\% der Fälle eine fortgeschrittene Neoplasie entdeckt, während in der Gruppe der 40- bis 49-Jährigen mit einer familiären Krebsbelastung in 3,4\% der Fälle eine fortgeschrittene Neoplasie nachgewiesen wurde. Des Weiteren wurde in einer britischen Studie mit 265 mindestens 45jährigen Patienten, bei denen neu rektale Blutungen aufgetreten waren, in 4,9\% der Fälle ein Adenom und in 5,7\% ein kolorektales Karzinom diagnostiziert.

Auf der Suche nach verlässlichen Methoden für den nichtinvasiven Nachweis von kolorektalen Karzinomen wurde in einer japanischen Studie mit 21.805 asymptomatischen Personen die Sensitivität des immunochemischen fäkalen Hämoccult-Tests ermittelt, die für fortgeschrittene Adenome $27,1 \%$ und für invasive Karzinome $65,8 \%$ betrug. Eine neue französische Studie mit 10.673 asymptomatischen Personen zeigte nun, dass der immunochemische fäkale Hämoccult-Test bezüglich der Identifikation von Karzinomen und vor allem von fortgeschrittenen Adenomen eine höhere Sensitivität besitzt als der herkömmliche fäkale HämoccultTest. Außerdem wurde mit dem immu- nochemischen fäkalen Hämoccult-Test in einer israelischen Studie, an der 1000 Individuen mit erhöhtem Neoplasierisiko oder Symptomen teilnahmen, für fortgeschrittene Adenome eine Sensitivität von $67 \%$ und eine Spezifität von $91,4 \%$ gezeigt, für Karzinome wurde sogar eine Sensitivität von $87,5 \%$ und eine Spezifität von $94,1 \%$ erreicht.

\section{Ösophaguserkrankungen}

Zur Behandlung der Achalasie ergab eine schwedische prospektive Studie mit 51 Patienten, dass bei der laparoskopischen Myotomie mit Hemifundoplication die Gesamtkosten trotz der initial größeren klinischen Effizienz signifikant höher waren als bei der pneumatischen Dilatation. Aufgrund der Ergebnisse von verschiedenen Studien liegt die Inzidenz des Plattenepithelkarzinoms bei der Achalasie im Bereich von 0,4-3,4 pro $1000 \mathrm{~Pa}$ tienten pro Jahr, das entspricht einem im Vergleich zu dem der allgemeinen Bevölkerung um das 3- bis 33fach erhöhten Risiko. Eine neue schwedische retrospektive Kohortenstudie mit 2896 an Achalasie leidenden Patienten zeigte nun, dass die standardisierte Inzidenzrate nicht nur für das Plattenepithelkarzinom, sondern auch für das Adenokarzinom über 10 liegt.

Die Kontroverse, ob es sich bei der gastroösophagealen Refluxkrankheit um ein Spektrum von ineinander übergehenden Krankheiten oder um eine kategorische Krankheit mit unabhängigen Entitäten handelt, wurde in einer großen Kohortenstudie mit 3894 an Sodbrennen leidenden Patienten aufgrund des Langzeitverlaufs der Erkrankung nach der Behandlung mit Esomeprazol untersucht. Nach einer Nachbeobachtungsdauer von zwei Jahren war die Erkrankung bei ungefähr $75 \%$ der Patienten mit einer initial nichterosiven Refluxkrankheit und bei $98 \%$ der Patienten mit einer erosiven Erkrankung vom Grad A oder B nicht fortgeschritten, wobei aber bei einem erheblichen Patientenanteil eine Progression sowie ein mit dem initialen Schweregrad der Krankheit zunehmendes Risiko für die Entwicklung eines Barrett-Ösophagus festgestellt wurden. Gemäß diesen Resultaten ist die Refluxkrankheit trotz des mehrheitlich stabilen Verlaufes keine kategorische Krank- 
heit, weshalb insbesondere bei einer Ösophagitis vom Grad C oder D eine regelmäßige endoskopische Überwachung angezeigt ist.

Zum Langzeitverlauf der chronischen Refluxkrankheit ergab eine Studie mit 298 Patienten nach einer Nachbeobachtungsdauer von 7 Jahren für die Antirefluxoperation eine größere Ansprechrate bezüglich anhaltender Symptomkontrolle als für die Behandlung mit Omeprazol, wobei aber die Operation mit einer erhöhten Inzidenz von Nebenwirkungen wie Dysphagie, Unfähigkeit aufzustoßen und rektaler Flatulenz einherging. Nach einer Metaanalyse von 10 Studien mit 15.316 Patienten mit einer erosiven Ösophagitis wurde unter der 8-wöchigen Behandlung mit Esomeprazol im Vergleich zu den Protonenpumpeninhibitoren Omeprazol, Lansoprazol und Pantoprazol eine signifikant höhere Heilungsrate erreicht. Dabei war die therapeutische Überlegenheit von Esomeprazol bei einer schweren Ösophagitis besonders ausgeprägt.

\section{Helicobacter pylori und Ulkuserkrankungen}

Die bei Patienten mit oberen gastrointestinalen Blutungen vor der endoskopischen Untersuchung vorgenommene intravenöse Verabreichung von hochdosiertem Omeprazol bewirkte im Vergleich zu Placebo eine signifikante Verringerung der notwendigen endoskopischen Therapien und ermöglichte für mehr Patienten eine Entlassung aus der stationären Behandlung innerhalb von 3 Tagen. Der Transfusionsbedarf, die Häufigkeit rezidivierender Blutungen, die Anzahl notfallmäßiger Interventionen und die 30-Tagesmortalität waren in beiden Gruppen ähnlich. Zur Frage nach der kosteneffizientesten Strategie bei der Behandlung von Ulkusblutungen zeigt eine Modellrechnung, dass sich bei der intravenösen Verabreichung im Vergleich zur oralen Einnahme eines Protonenpumpeninhibitors $\mathrm{Zu}$ satzkosten von mehr als 700.00o US-Dollar pro gewonnenem qualitätskorrigierten Lebensjahr ergeben. Die orale Einnahme einer neuen Formulierung von Esomeprazol mit einer Natriumbikarbonat-Hülle führte im Mittel bereits nach 2 Minuten zu einer Anhebung des intragastralen $\mathrm{pH}$ -
Wertes auf $>6$, bei der intravenösen Verabreichung von Pantoprazol wurde dies erst nach 9o Minuten erreicht.

Im Zusammenhang mit den Diskussionen um die kardiovaskuläre Verträglichkeit der antirheumatischen Behandlung ergaben die Resultate von zwei Metaanalysen, dass das Risiko für ein vaskuläres Ereignis nicht nur unter der Behandlung mit einem selektiven Cyclooxygenase-2Inhibitor, sondern auch unter derjenigen mit den klassischen nichtsteroidalen Antirheumatika Diclofenac und Ibuprofen erhöht ist. Im Einklang mit diesem Ergebnis wurde in der MEDAL-Studie bei den 34.701 teilnehmenden Patienten mit einer Arthrose oder einer chronischen Polyarthritis unter der Therapie mit dem selektiven Cycloxogenase-2-Inhibitor Etoricoxib nach einer mittleren Behandlungsdauer von 18 Monaten ein ähnlich hohes Risiko für ein kardiovaskuläres Ereignis festgestellt wie unter der Therapie mit dem klassischen nichtsteroidalen Antirheumatikum Diclofenac. Allerdings war die Wahrscheinlichkeit für ein Ereignis im oberen Gastrointestinaltrakt bei den mit Etoricoxib behandelten Patienten signifikant niedriger als bei den Patienten der Diclofenac-Gruppe, wogegen die Anzahl der klinischen Ereignisse im unteren Gastrointestinaltrakt unter der Therapie mit Etoricoxib im selben Bereich lag wie unter der Behandlung mit Diclofenac.

\section{Chronisch-entzündliche Darmerkrankungen}

Patienten mit einem M. Crohn haben in den ersten Jahren nach einer Ileozökalresektion ein relativ hohes Rezidivrisiko, das gemäß einer belgischen Doppelblindstudie mit 81 Patienten durch die 3-monatige Kombinationstherapie mit Metronidazol und Azathioprin im Vergleich zu Placebo signifikant gesenkt wird. Eine Verbesserung des postoperativen Verlaufs wurde auch in einer retrospektiven Studie mit 579 Patienten mit einem M. Crohn gezeigt, bei denen nach einer Darmoperation die Anzahl der notwendigen Reoperationen durch die postoperative Behandlung mit Azathioprin oder 6-Mercaptopurin signifikant verringert wurde.

Bei der Induktionstherapie des steroidabhängigen aktiven M. Crohn führ- te die zusätzlich zu Azathioprin oder 6Mercaptopurin erfolgende Verabreichung von Infliximab einer französischen Studie mit 113 Patienten zufolge nach 12, 24 und 52 Wochen zu einer im Vergleich mit Placebo signifikant höheren Remissionsrate, wobei die Patienten ohne vorgängige Behandlung mit Azathioprin oder 6-Mercaptopurin einen größeren Nutzen aus der Kombinationstherapie zogen als die bereits mit diesen Immunmodulatoren Behandelten. Demgegenüber zeigte eine retrospektive Auswertung der Daten der klinischen Studien ACCENT I und II sowie ACT I und II, dass bei der Erhaltungstherapie von chronisch-entzündlichen Darmerkrankungen durch zusätzlich zu Infliximab gegebene Immunmodulatoren keine wesentliche Verbesserung der Ansprech- und Remissionsraten erreicht werden kann.

Bezüglich der Wirksamkeit der Erhaltungstherapie bei chronisch-entzündlichen Darmerkrankungen ergab die französische Doppelblindstudie CHARM, dass bei den Patienten mit einem mittelschweren bis schweren M. Crohn, die auf eine 4-wöchige Induktionstherapie mit Adalimumab angesprochen hatten, durch die Fortsetzung der Behandlung mit Adalimumab während 56 Wochen die Remission im Vergleich zu Placebo bei einem signifikant größeren Anteil erhalten werden konnte. Mit den Wirkstoffen Infliximab, Adalimumab und Certolizumab, die einer Metaanalyse zufolge bei der Behandlung des M. Crohn eine nahezu gleiche Wirksamkeit besitzen, wird auch auf dem Gebiet der Anti-Tumornekrosefaktor- $\alpha$ eine individualisierte Behandlung möglich werden.

\section{Korrespondenzadresse \\ Prof. Dr. M. Fried}

Klinik für Gastroenterologie und Hepatologie, UniversitätsSpital Zürich

Rämistrasse 100, 8091 Zürich

michael.fried@usz.ch

Interessenkonflikt. Keine Angaben Das vollständige Literaturverzeichnis .. ... finden Sie in der html-Version dieses Beitrags im Online-Archiv auf der Zeitschriftenhomepage www.DerGastroenterologe.springer.de 